\title{
NOISE INVESTIGATIONS ON THICK FILM RESISTORS ${ }^{\dagger}$
}

\author{
A. AMBROZY \\ Technical University, Budapest, Hungary \\ and \\ G. WOLLITZER \\ Microelectronics Company, Budapest Hungary \\ (Received November 1, 1982; in final form July 6, 1983)
}

Compared to metallic thin films or wires thick film resistors have a rather complex structure. Transition metal oxide particles are embedded into a glassy matrix. The resistivity depends heavily on the percentage of the constituents in the composition. It is believed that the main part of the resistance stems from the touching areas of conductive grains and that the conduction is by tunneling.

To give a better insight a noise spectrum analysis has been developed. A DC current is passed through the sample to be measured. The output noise voltage is analyzed between $1.6 \mathrm{~Hz}$ and $25 \mathrm{kHz}$ using a commercial third-octave analyser.

Results are given of an experiment in which the glassy matrix of thick film resistors was etched away step by step and the noise was measured vs. the number of etching steps.

\section{INTRODUCTION}

Excess noise (often called "current noise") has been observed in all types of non-pure metallic resistors, including thick film resistors. The power spectrum of this noise may be expressed in the following general form:

$$
S(f)=\text { const } \frac{I^{\beta}}{f^{\alpha}}
$$

where I is the DC current flowing through the device, f frequency of measurement, $\beta \approx 2$ and $\alpha \approx 1$. The current dependence may be easily interpreted as the fluctuation of the conductance or resistance. ${ }^{3}$

The structure of a thick film resistor is very complex. Conducting metal oxide grains are embedded into a glassy matrix. While at low resistivities the grains probably touch each other and the resulting resistivity is determined by constriction resistances, at high resistivities the main factor is the doped glassy matrix. There is a strong belief ${ }^{2}$ that the main conduction mechanism is the tunneling.

\section{NOISE MEASUREMENTS}

The noise measuring setup is shown in Figure 1. In series with the resistor $R_{m}$ to be investigated there is an excess-noise-free (wiresound) resistor of 100 kiloohm. The DC

\footnotetext{
${ }^{\dagger}$ Paper originally presented at the 5th International Spring Seminar on Electrotechnology held at Prenet Czechoslovakia, 1-4 June, 1982.
} 


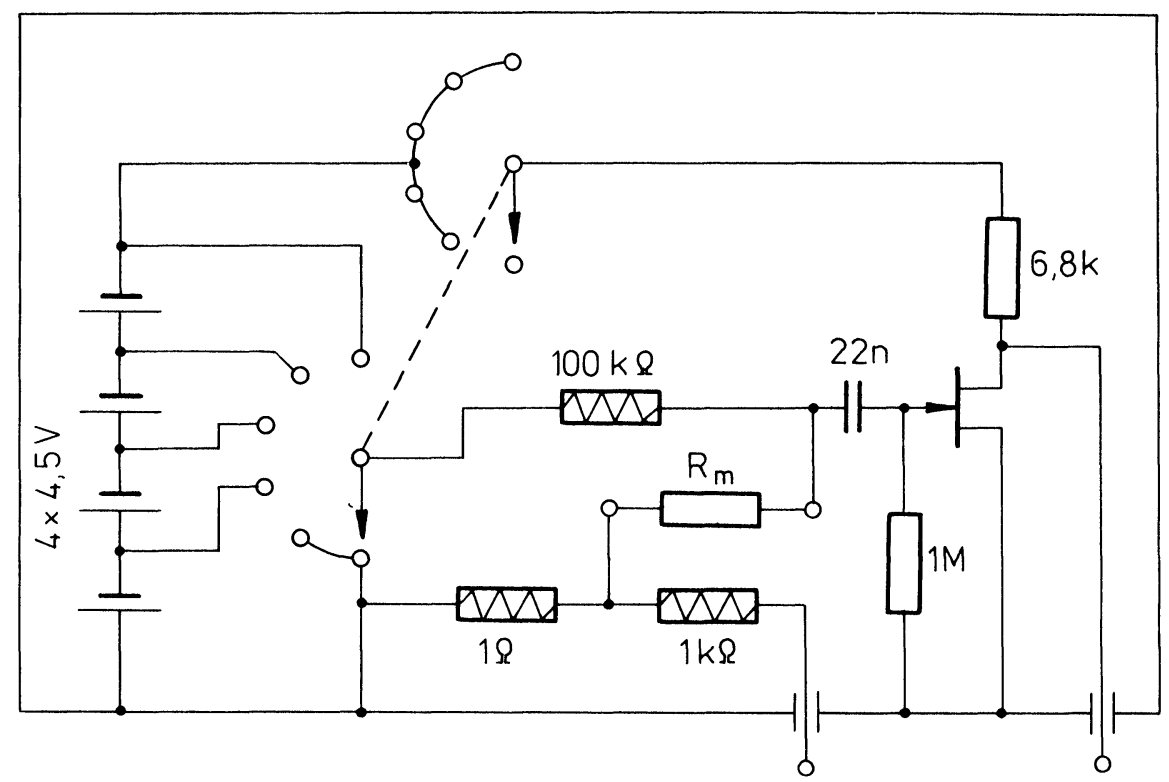

FIGURE 1 Noise measuring setup.

current flowing through $R_{m}$ is provided by four standard batteries $4,5 \mathrm{~V}$ each. The total squared noise voltage across $R_{m}$ is

$$
u^{2}=4 k T\left(R_{s} \| R_{m}\right) \Delta f+k^{2} I^{2} R_{m}^{2} \frac{R_{s}^{2}}{\left(R_{m}+R_{s}\right)^{2}} \frac{\Delta f}{f}
$$

where $R_{S}$ is the series resistor, $k$ a proportionality factor, $I$ the DC current flowing through $R_{m}$. Since the latter is $I=U_{D C} /\left(R_{s}+R_{m}\right)$

$$
\mathrm{u}^{2}=4 k T\left(R_{\mathrm{s}} \| R_{\mathrm{m}}\right) \Delta f+\mathrm{k}^{2} \mathrm{U}_{\mathrm{DC}}^{2} \frac{\mathrm{R}_{\mathrm{m}}{ }^{2} \mathrm{R}_{\mathrm{s}}{ }^{2}}{\left(\mathrm{R}_{\mathrm{m}}+\mathrm{R}_{\mathrm{s}}\right)^{4}} \frac{\Delta \mathrm{f}}{\mathrm{f}}
$$

where the first term is the thermal noise and the second the excess one. It has a maximum when $\mathrm{R}_{\mathrm{m}}=\mathrm{R}_{\mathrm{s}}$; moreover the JFET preamplifier produces the least noise if $R_{\mathrm{m}} \| \mathrm{R}_{\mathrm{s}} \approx 50 \mathrm{k} \Omega$. So this setup provides the best results with $\mathrm{R}_{\mathrm{m}}=30 \ldots 200$ kiloohms.

For the order of $3 \ldots 30$ kiloohms a bipolar transistor preamplifier is the proper selection.

The output of the preamplifier is connected to a main amplifier with a gain of 1000 . Both units are battery operated and enclosed in steel boxes. The amplified noise signal was analyzed formerly by a home made active filter having 9 bands between $10 \mathrm{~Hz}$ and $10 \mathrm{kHz}$ and was detected by a time varying integrator. ${ }^{3}$ Now, the latter units are replaced by a real-time spectrum analyser of type Brüel-Kjaer 2131 which works within $1,6 \mathrm{~Hz}$ and $20 \mathrm{kHz}$ providing 41 third-octave bands. A typical noise spectrum of a thick film resistor is shown in Figure 2. 


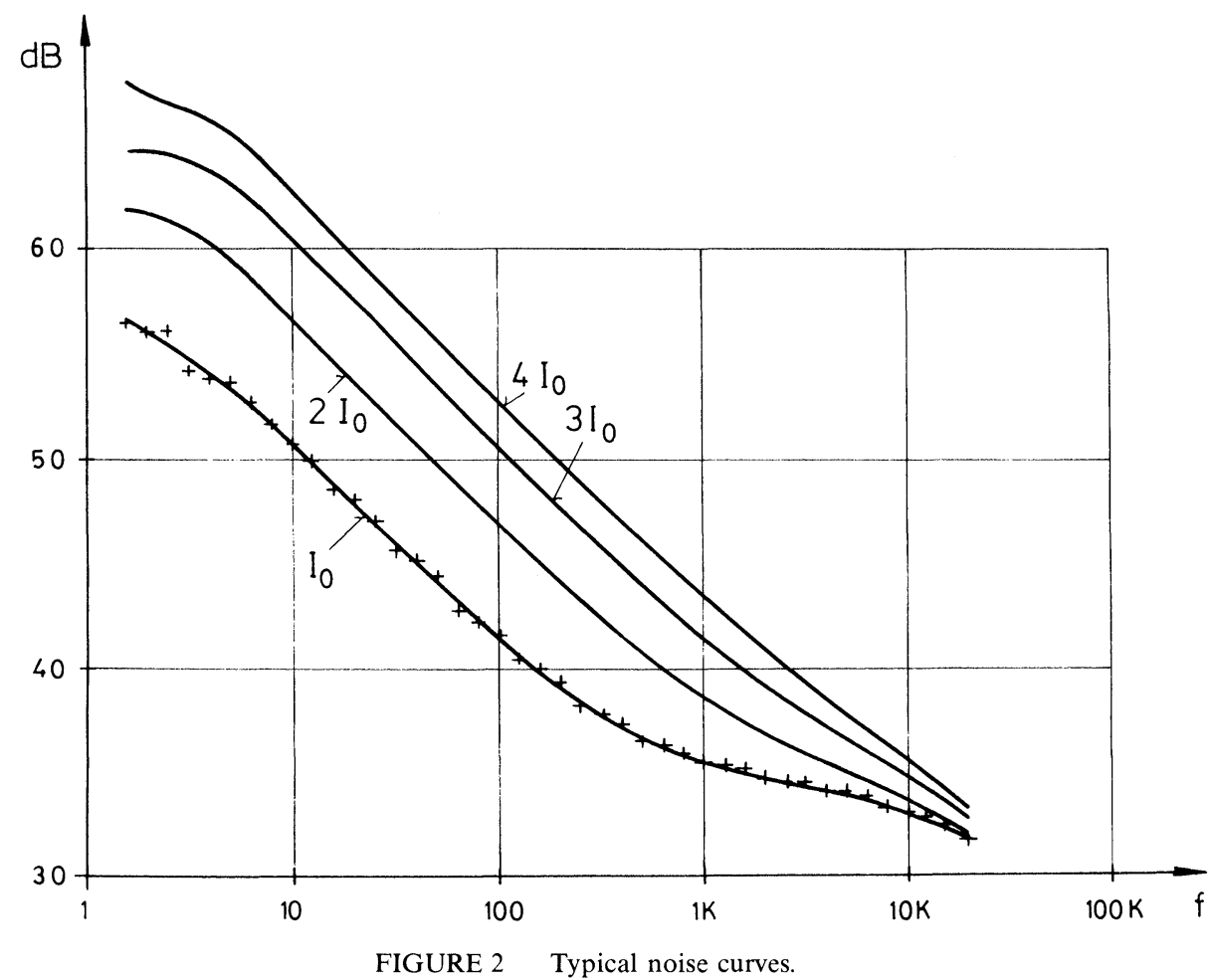

\section{SAMPLE PREPARATION}

Samples were made on $96 \%$ alumina substrate using a ternary conductor ink (PdPtAg, type REMEX 2014) and a ruthenium-oxide resistor ink of 100 kiloohm per square (REMEX 8051). The samples were fired at $850^{\circ} \mathrm{C}$ for eight minutes while the total cycle continued for 65 minutes. As we will see later the repeated firing (refiring) is an important element of our investigations. All subsequent refirings were done under the above circumstances.

\section{INVESTIGATIONS ON SAMPLES}

The role of the glassy matrix has been investigated by partial etching. The samples were etched in a dilute water solution of $0,05 \%$ fluoric acid and $0,01 \%$ nitric acid for a period of $0 . .16$ minutes and subsequently they were immediately rinsed by deionized water as well as methanol and finally they were dried in $100^{\circ}$ air. The composition of the etchant and the time was set to obtain a well-observable dissolving effect but without making coarse damages on resistors.

Figure 3 shows the noise factor (in arbitrary units) vs. etching time. It is interesting that two valleys are superimposed on a monotonous curve.

The resistance of the samples increased in response to etching. This was almost linear with a slope of 2,4 per cent per minute. The increase can be explained by the fact that a part of the resistor body (the poor conducting glass) is removed. 


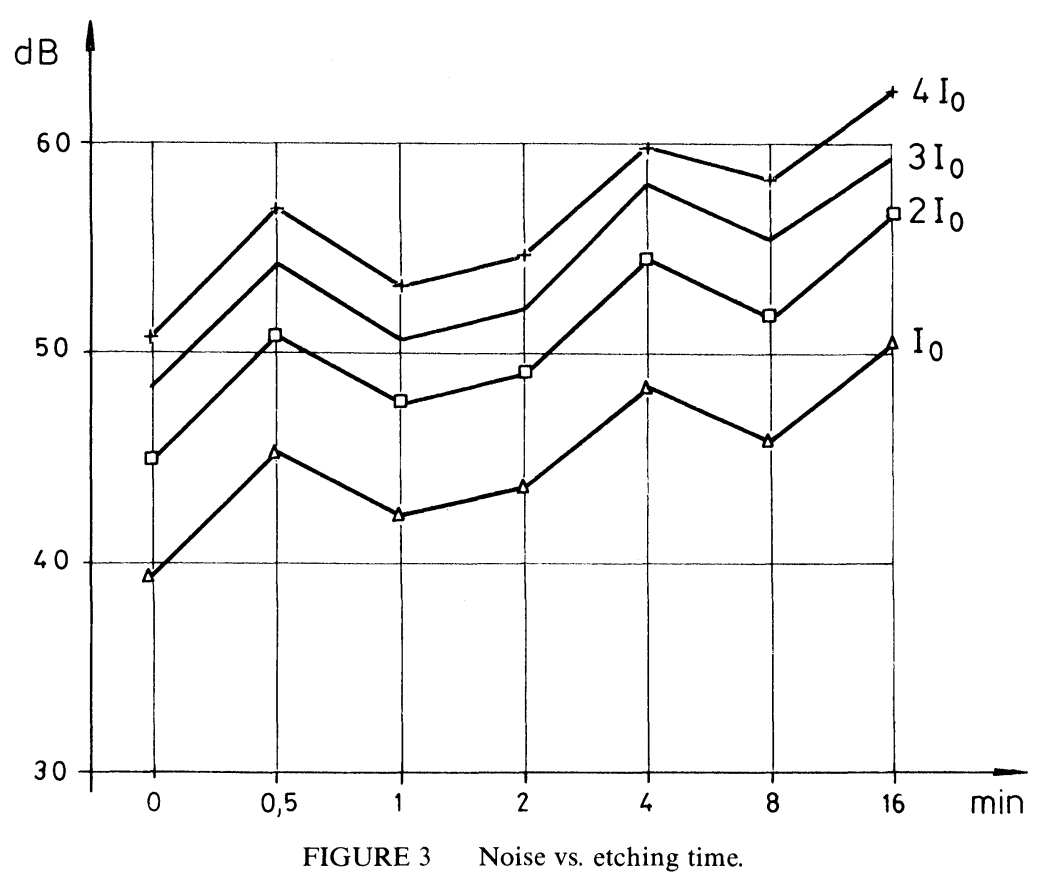

To stabilize the etched resistors we applied 1-3 refirings. The change in resistance - which is always negative - is shown in Figure 4. The loss of glass probably

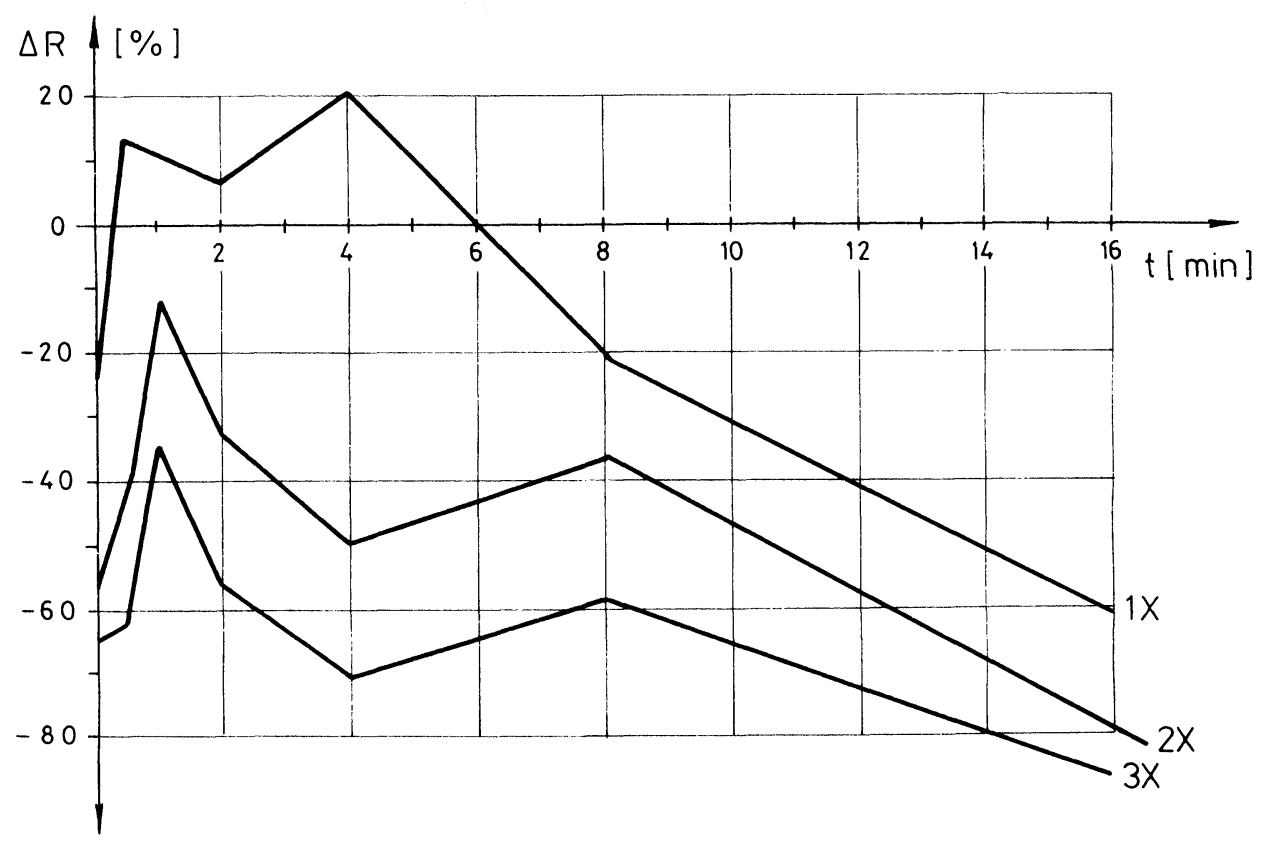

FIGURE 4 Resistance change vs. etching time. 
results in more directly touching ruthenium oxide grains. What is more interesting is that the maxima of Figure 4 coincide with minima of Figure 3. No explanation is available at present.

Similar resistance-etching time characters have been found on 1 and 10 kiloohm per square resistors (8031 and 8041). On the other hand, refiring of unetched resistors resulted in always decreasing resistance.

While the experiments described above gave only initial results we hope that combining them with another microanalytical investigations (say electron microprobe) we will understand better the basic properties of thick film resistors.

\section{REFERENCES}

1. A. Ambrózy, $1 / \mathrm{f}$ noise in resistors made of grainy material". Proceedings of the 4.International Spring Seminar on Electronics Technology, Ilmenau, 114-119 (1980).

2. G.E. Pike, C.H. Seager, "Electrical properties and conduction mechanisms of Ru based thick-film resistors". J. Appl. Phys. 48, 5152-5169 (1977/12).

3. A. Ambrózy, "Reducing the time requirement in direct reading noise measurement" Proc IEEE. 53, 1161$1162(1965 / 8)$. 

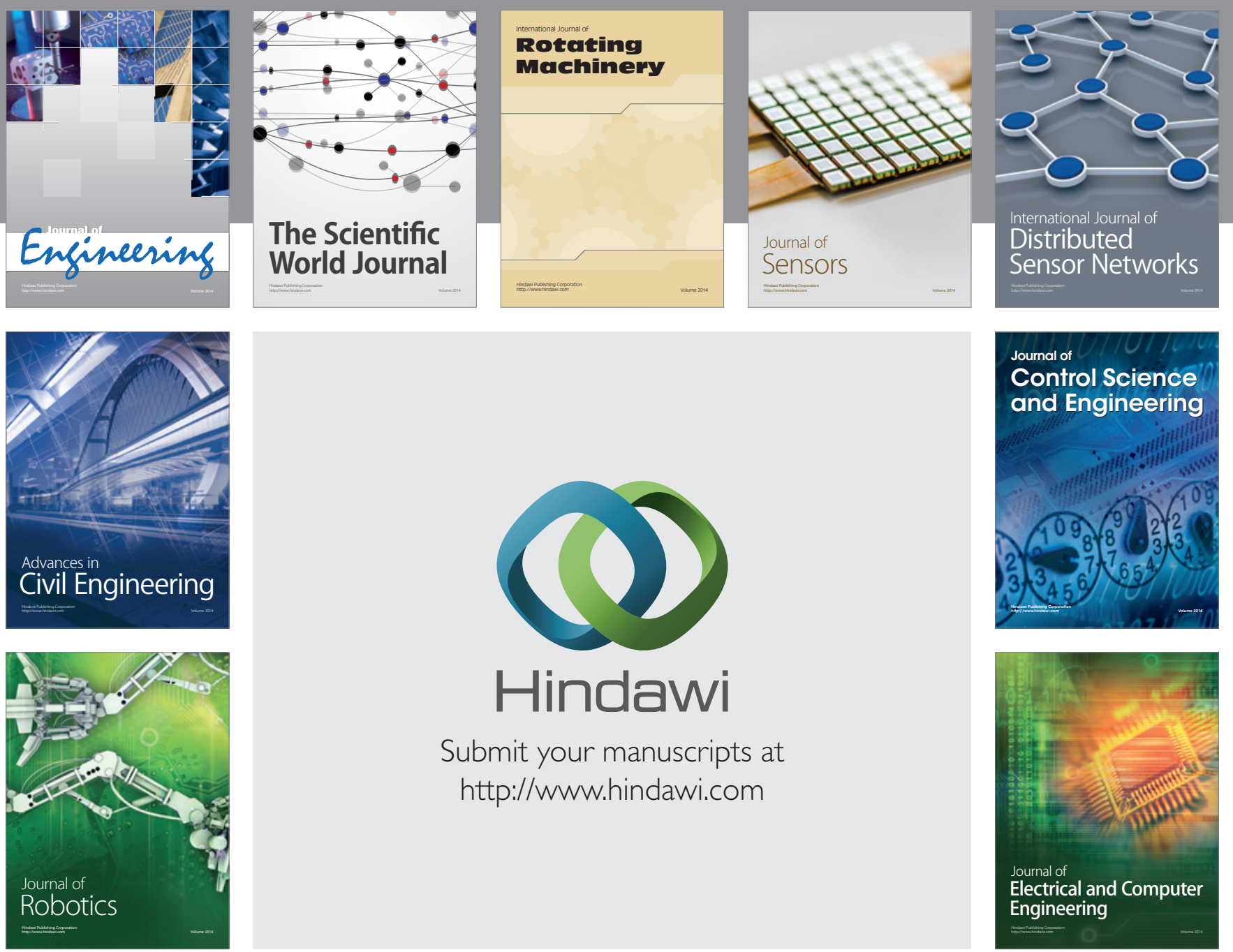

Submit your manuscripts at

http://www.hindawi.com
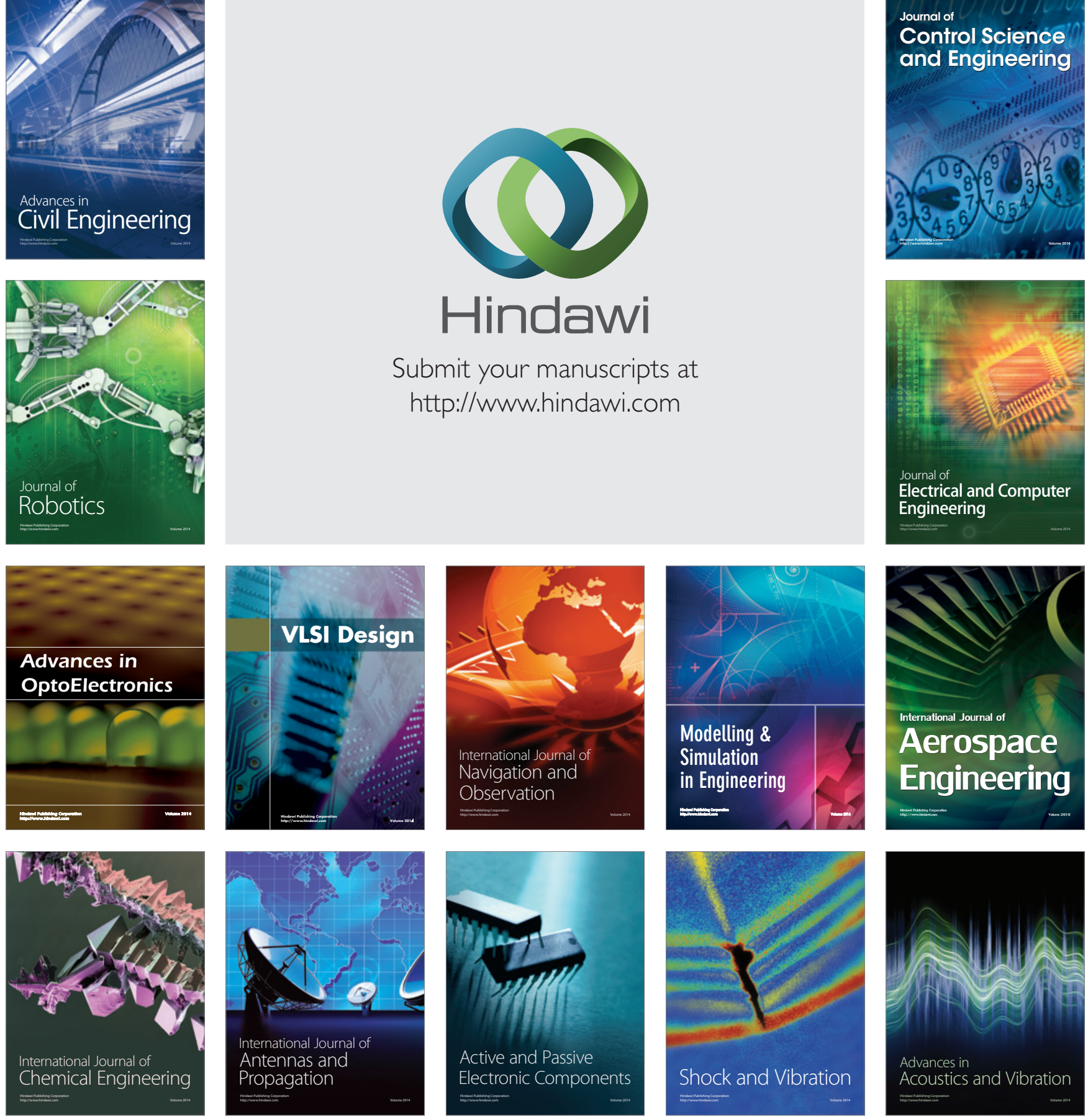